number of the victims and of that the incident occurred in a weekday morning.

Prehosp Disaster Med 2011;26(Suppl. 1):s154-s155

doi:10.1017/S1049023X11005024

\section{(P2-59) Monocular Vision Loss Following Blunt Trauma} P.M. Pustinger, D. Paratore

College of Osteopathic Medicine, East Lansing, United States of America

Blunt trauma is a leading cause of injury in the teenage population. The early detection of injury is the primary goal of emergency medicine in order to maintain an optimal functional capacity. This is of particular importance in the pediatric population. The following is a case presentation of monocular vision loss in a 14-year-old girl as a result of traumatic optic neuropathy. A motor vehicle collision was the cause of injury for this patient. She was an unrestrained rear seat passenger and struck her head on the driver's headrest during a frontal impact. A delayed presentation of over seven hours added to the complexity of this presentation. Further, a non-contrasted computed tomography (CT) scan of the head and orbits was unremarkable except for soft tissue swelling. The child was left with only light perception in the affected eye. This case presentation will illustrate the importance of immediate care, diagnostic studies, proper consultant input, follow-up care, and the natural history of the injury for this most unusual case.

Prehosp Disaster Med 2011;26(Suppl. 1):s155

doi:10.1017/S1049023X11005036

\section{(P2-60) Thyroid Storm in the Emergency Department}

\section{M.J. Van veelen, ${ }^{1}$ L. Yurtsever, ${ }^{2}$ M. Baggen, ${ }^{1}$}

E.A. Dubois ${ }^{3}$

1. Department of Internal Medicine, Rotterdam, Netherlands

2. Rotterdam, Netherlands

3. Cardiology, Rotterdam, Netherlands

Case: A 20-year-old woman was referred to the emergency department with rapid acceleration of complaints of palpitations, fever, diarrhea, and agitation that had been present for several weeks. During physical examination, the patient was uncomfortable and restless with a tachycardia of $170 /$ minute, and a fever of $38.5^{\circ} \mathrm{C}$. Palpation of the neck revealed a small ventral, painless, solid elastic mass, more prominent on the right side, clinically suspicious for goiter. An electrocardiograph showed an atrial flutter of $150 / \mathrm{min}$. Initial laboratory results showed an erythrocyte sedimentation rate of $35 \mathrm{~mm} /$ hour $(0-20 \mathrm{~mm} /$ hour $)$ and urine analysis tested positive for ketones.

Outcome and Treatment: The patient was presumed to be suffering from a thyroid storm. She was treated promptly with Propranolol $160 \mathrm{mg}$ and Thiamazole $30 \mathrm{mg}$ twice daily at the emergency department. She was admitted to the Cardiac Care Unit for observation of the heart rhythm, which slowed down to $110 /$ minute the same day and her condition improved clinically. The following day her laboratory result confirmed the diagnosis with a thyroid-stimulating hormone of $<0.01$ $\mathrm{mIU} / \mathrm{L}(0.4-4.0 \mathrm{mIU} / \mathrm{L})$ and a free thyroxine $(\mathrm{T} 4)$ of $>75$ pmol/1 (10-22 pmol/1). Eventually, she was diagnosed with Graves Disease.

Discussion: Thyroid storm is an acute, life-threatening, hypermetabolic state induced by excessive release of thyroid hormones. The adult mortality rate is high (90\%) if early diagnosis is not made and the patient is left untreated. Therefore, in case of clinical suspicion for thyroid storm, it is critical to start prompt treatment with Beta blockade and Thiamazole before the diagnosis can be confirmed biochemically.

Prehosp Disaster Med 2011;26(Suppl. 1):s155

doi:10.1017/S1049023X11005048

(P2-61) Hiccups with Chapman/Carberra Sign And left Bundle Branch Block in Anterior Wall Myocardial Infarction

D. Mishra, D.T. Koli

Emergency Department, New Delhi, India

Patients with myocardial infarction can present to the Emergency Department with atypical symptoms. A 60-year-old male presented with a fever for two days and ongoing hiccups he had experienced for four hours. He also had experienced an episode of vomiting. An electrocardiograph revealed ascending limbs of the S-waves in leads V3/V4, notching $>0.05$ seconds in the ascending limbs of the R-waves in leads I, aVL, V6, and the presence of a left bundle branch block.

Keywords: emergency department; fever; hiccups; left bundle branch

block; myocardial infraction

Prehosp Disaster Med 2011;26(Suppl. 1):s155

doi:10.1017/S1049023X1100505X

(P2-62) An Infrequent Case of Orthopedic Emergencies Open Dorsal Dislocation of the Proximal Interphalangeal (PIP) Joint Dislocation

A. Bayir, ${ }^{1}$ U. Kaldirim, ${ }^{1}$ S. Ardic, ${ }^{2}$ Y.E. Eyi ${ }^{1}$ I. Arziman, ${ }^{1}$

M. Durusu ${ }^{1}$

1. Department of Emergency Medicine, Etlik - Ankara, Turkey

2. Emergency Medicine, Ankara, Turkey

Introduction: Reducible open dorsal dislocation of the single finger's PIP joint is an infrequent case of orthopedic emergencies. The severity of this injury may be underestimated. These injuries are associated with long-term complications such as synovitis, stiffness, degenerative arthrosis, septic arthritis, and loss of the digit if suboptimally treated.

Case: A 90-year-old male came to the emergency department with an open dorsal PIP dislocation due to a fall on his right hand. There was a transverse skin laceration just proximal to the PIP flexion crease of his small and ring fingers. The condyles of his small finger's proximal phalanx protruded through the wound. X-rays showed a dorsal dislocation of the PIP joint without fracture. There was no neurovascular injury determined. The proximal phalanx was hyperextended slightly with gentle axial traction. After irrigation, the skin wound was closed primarily without repair of damaged structures, and systemic antibiotherapy was performed for a week. The PIP joint was immobilized for three weeks by applying the splint dorsally with the joint in 20 degrees of flexion. Active range of motion exercises were then implemented, and the patient regained full digital flexion with only a 10 degree loss of extension within eight weeks.

Discussion: Forced hyperextension with axial compression causes a dorsal dislocation of the PIP joint. Dorsal PIP dislocations are more common than volar IP dislocation. 